\title{
Autoestima como eXPREssão de saúde MENTAL E DISPOSITIVO DE MUDANÇAS NA CULTURA ORGANIZACIONAL DA POLÍCIA
}

\author{
Edson Ribeiro de Andrade* \\ Edinilsa Ramos de Souza ${ }^{* *}$
}

\section{ResUMo}

O presente artigo focaliza parte de uma pesquisa-ação com policiais civis do Rio de Janeiro. O objetivo foi problematizar como a autoestima e a saúde mental desses profissionais afetam e são afetadas pela cultura organizacional da polícia. Buscou-se demonstrar que o resgate da autoimagem pode agir como ferramenta eficaz de mudanças na vida pessoal e profissional desses profissionais e da própria instituição. Para isto, foram aplicados questionários e técnicas qualitativas em 148 policiais (76 no Grupo Experimental e 72 no Grupo Controle) antes, durante e após a intervenção. Conclui-se que a pesquisa foi uma contribuição importante em nossa realidade, constituindo uma intervenção sistematizada com policiais.

Palavras-chave: polícia; autoestima; cultura organizacional; saúde mental.

\section{Abstract}

Self-ESTEEM AS an EXPRESSion OF MENTAL HeALTH AND DEVICE CHANGES IN ORGANIZATIONAL CULTURE OF THE POLICE

The present article focuses on part of a previous action research with police officers in Rio de Janeiro. We aimed to discuss how self-esteem and mental health professionals of these affect and are affected by the organizational culture of the Police. We sought to demonstrate that the

* Doutorando em Saúde Pública da ENSP/Fiocruz. Professor e coordenador do PósGraduação de Saúde Coletiva e Recursos Humanos do ISECENSA.

** Doutora em Saúde Pública pela Fundação Oswaldo Cruz, pesquisadora associada da Fundação Oswaldo Cruz. 
recovery of self-image can act as an effective tool for change in personal and professional lives of these professionals and the institution itself. For this, we applied questionnaires and qualitative techniques in 148 police officers ( 76 in the experimental group and 72 in the control group) before, during and after the intervention. We concluded that the research was a major contribution in our reality, constituting a systematic intervention with the police.

Keywords: police; self-esteem; organizational culture; mental health.

\section{INTRODUÇÃO}

Este artigo apresenta dados de uma pesquisa cujo principal objetivo foi fornecer suporte emocional a policiais civis da cidade do Rio de Janeiro, garantindo, assim, o direito à saúde mental. Foram abordados quatro constructos: autoestima, qualidade de vida, cultura e clima organizacional. Andrade, Sousa, e Minayo (2009) refletiram acerca dos impactos da referida intervenção na autoestima dos policiais e na qualidade de vida destes. Neste artigo buscou-se discutir, a partir da literatura e dos resultados da intervenção, as influências da cultura organizacional da polícia sobre a autoestima dos agentes.

Para alguns estudiosos, a cultura organizacional da polícia pode ser considerada uma cultura de violência (Adorno, 1999; Mesquita Neto, 1999). Fato este que pode causar, dependendo da atitude de cada agente, profundos danos à saúde destes trabalhadores. A tese subjacente a esta proposta é a de que as forças de segurança pública pouco valorizam a subjetividade dos seus agentes nas propostas políticas e gerenciais, se apoiando em tecnologias de ação e no corporativismo organizacional.

Parte dos crimes contra o patrimônio que ocorrem na cidade do Rio de Janeiro são investigados na delegacia em que se desenvolveu o estudo. Os policiais que nela atuam não têm como missão precípua investigar crimes contra a pessoa, mas nem por isso deixam de sofrer estresse e pressão da sociedade, que lhes atribui a tarefa de protegê-la da criminalidade e garantir a segurança dos cidadãos e do seu patrimônio. Nesse sentido, a vida emocional e afetiva do policial tem pouca ou quase nenhuma importância. Pretende-se que ele haja como "máquina" e cumpra seu papel de investigar e prevenir a criminalidade, sem deixar que suas emoções ou "fraquezas" sentimentais afetem seu desempenho.

Talvez por isso sejam escassos os estudos em nossa realidade acerca dos policiais enquanto sujeitos de direitos, dentre os quais estão: expressar sua subjetividade e viver suas emoçôes. Um dos poucos estudos brasileiros que abordaram aspectos da vida do policial foi o de Minayo e Souza (2003). Nele, as autoras, traçaram um 
amplo panorama das condições de trabalho, de saúde e da qualidade de vida dos policiais civis da cidade do Rio de Janeiro. Elas utilizaram uma amostra representativa de cerca de 2.700 agentes. Nessa investigação, constatou-se que a vida emocional do policial civil é atravessada por um somatório explosivo de sentimentos, dentre os quais: risco permanente de sofrer agressōes, desmotivação, desesperança, revolta por sentir-se pouco valorizado e, às vezes, injustiçado por seus superiores hierárquicos e pela sociedade.

As autoras ressaltam que um servidor que atua em um regime de "insalubridade emocional”, como o observado entre os agentes de segurança do país, particularmente os do Rio de Janeiro, deveria ter direito a um suporte emocional para enfrentar o seu dia-a-dia. Caso contrário podem atingir um grau de esgotamento psíquico que compromete sua vida profissional e pessoal. As condições de trabalho insalubres imputam-lhes um grau de sofrimento que, segundo a psicodinâmica do trabalho, pode lhes causar ruptura do equilíbrio psíquico. Essas autoras defendem, ainda, um acompanhamento psicológico específico para os policiais civis, principalmente para os que atuam em atividades operacionais, ou seja, na linha de frente nas delegacias de polícia. "Consideramos ser urgente a criação de uma instância específica para apoio psicológico dos policiais” (Minayo e Souza, 2003: 333). E acrescentam a necessidade de criação de ações direcionadas à capacitação dos policiais "com ênfase na sua motivação e no seu bem-estar" (Minayo e Souza, 2003: 338).

Além das questôes abordadas, outras peculiaridades foram identificadas na referida pesquisa. Destacamos três pontos que merecem, aqui, especial menção: (1) metade dos policiais não tirava férias há mais de três anos; (2) a polícia civil funciona com $47 \%$ do contingente necessário; (3) os policiais estão em contato com a morte, inclusive de seus colegas, quase diariamente. Os itens acima demonstram a necessidade premente de ações afirmativas a favor da pessoa dos policiais civis. Segundo Minayo e Souza (2003), essas ações devem representar acolhimento, visar o seu bem-estar e direito ao suporte emocional a fim de que possam desempenhar adequadamente as suas funções.

Outro pressuposto da pesquisa, que embasa o presente trabalho, é o de que os policiais só podem cumprir efetivamente seu papel de agentes de segurança com a melhora da sua autoestima. Esta se encontra negativamente afetada pela pouca valorização da sua subjetividade pela corporação, pela visão negativa que a sociedade tem deles e pela consciência que têm dessa situação.

Dejours (1999) faz uma estreita relação entre o sofrimento psíquico dos trabalhadores e as relações que estes estabelecem nos espaços profissionais. Afirma que, se a ambiência é boa, as tarefas difíceis são percebidas de formas estimulado- 
ras. Por outro lado, ambientes ruins de trabalho favorecem estímulos negativos ao trabalho.

Nesta mesma direção, Minayo e Souza (2003) destacam a existência de algumas diferenças da Polícia Civil em relação aos componentes mais fortemente hierarquizados da Polícia Militar. E, apesar de amenizados na primeira pelo advento dos concursos públicos e das delegacias legais, "os conflitos são constantes e se apresentam, sobretudo, nas formas tradicionais de 'mando' e de gestão do trabalho" (Minayo e Souza, 2003: 162). Essa hierarquia, que obriga a disciplina sem questionamento, não contempla o indivíduo e sua subjetividade e influencia significativamente a autoestima destes profissionais.

Outra constatação importante advinda do diálogo com a literatura é que a cultura organizacional - entendida como formas de lidar com os problemas conforme conceitua Schein (1984) -, no caso policial, está impregnada de uma legitimação policial e institucional de práticas violentas, materializada por um estado de polícia que na verdade é um estado de guerra. Conforme Silveira Filho (2007), isto também tem influência sobre a autoestima dos operadores da segurança pública em nossa sociedade.

Este artigo tem como objetivo apresentar parte dos resultados de uma pesquisa que utilizou uma estratégia de resgate da autoestima, visando a transformação da cultura organizacional da Polícia.

\section{DEFINIÇÃo DE TERMOS}

O conceito de autoestima tem extrema relevância na Psicologia, apesar de não haver consenso na literatura em torno de sua definição. Dolan (2006) destaca que a autoestima é um dos conceitos psicológicos mais utilizados atualmente, provavelmente pelo seu aspecto prático na compreensão da busca de felicidade por parte das pessoas.

A introdução do termo é atribuída a William James no ano de 1885, quando ele assim se refere: "o que sentimos por nós mesmos é determinado pela proporção entre nossas realizações e nossas supostas potencialidades; uma fração cujo denominador são nossas pretensões e o numerador, os nossos sucessos" (James, 1974: 200).

Branden (1995) discorda dessa posição. Para ele a autoestima é algo que vem de dentro do indivíduo. Está, portanto, ligada às suas operações mentais e não às circunstâncias externas bem ou malsucedidas. Assim, associar a autoestima de uma pessoa a fatores externos é propiciar o não crescimento. Essa foi a postura 
que norteou a intervenção realizada, pois o trabalho com os policiais foi feito independente das condições de trabalho em que estavam inseridos.

Ainda para Branden (1994), a maneira pela qual nos percebemos repercute em nossas ações, na vida profissional e pessoal. Esse autor também afirma que existe uma relação entre nossas reações e o que pensamos de nós mesmos. E enfatiza que "desenvolver a autoestima é expandir nossa capacidade de ser feliz" (Branden (1994: 11).

Staerke (1996: 77) defende que a autoestima "é um constructo, ou seja, ela é construída, ela é uma conquista pessoal, inalienável e intransferível”. A autora pontua que "pessoas de autoestima saudável também sofrem e sentem ansiedade" (Staerke, 1996: 78). Entretanto, não paralisam.

Já Dejours (1999) destaca a importância do reconhecimento para todos aqueles que trabalham. Minayo e Souza (2003) afirmam que o reconhecimento pode fazer com que o policial tenha maior motivação e, assim, enfrente o estresse que sua função exige. Por inferência, pode-se dizer que a desvalorização do trabalho policial causaria uma série de danos a esse grupo. As autoras citadas afirmam que: "O fato de realizar uma tarefa de opressão ao crime, assim como de existir na opinião pública a ideia de que o policial é violento e corrupto, conduz à percepção de um sentimento de injustiça e de desvalorização por parte da categoria" (Minayo \& Souza, 2003: 165).

Njaine et al. (2006) discutem que alguns sentem orgulho de ser policial pelo poder associado à sua profissão; outros sentem vergonha por saber que possuem uma imagem negativa associada à corrupção e truculência. Gaulejac (2006), refletindo sobre esse sentimento de vergonha, afirma que ele se instala quando a identidade da pessoa é alterada. Esse autor teoriza sobre os processos de estigmatização:

As referências habituais que lhe permite situar-se em relação aos outros e a si mesmo são fragilizadas ou destruídas. Em todos os casos, a autoestima é posta em questão pela falta de estima dos outros. O sujeito é dilacerado por tensões contraditórias entre a tentativa de salvaguardar sua unidade e a impossibilidade de consegui-lo sem rejeitar uma parte de si mesmo. Defronta-se com uma negação daquilo que constitui o todo ou parte de seu ser profundo. Este dilaceramento produz uma conflagração psíquica (Gaulejac, 2006: 103).

Nesse sentido, a conflagração psíquica significa sofrimento, isto é, o dilaceramento da identidade causa sofrimento. Balestreri (2003) afirma que é muito importante, do ponto de vista pessoal e para a sociedade, que o agente de segurança tenha orgulho de ser policial. Entretanto, isso só pode acontecer aprendendo-se o lugar existencial desta profissão. 
O policial, pela natural autoridade moral que porta, tem o potencial de ser o mais marcante promotor dos Direitos Humanos, revertendo o quadro de descrédito social e qualificando-se como um personagem central da democracia (Balestreri, 2003: 1). Branden (1994) também sugere que há uma relação entre a autoestima e uma atitude de cuidado com o outro. Esse autor afirma que quanto maior é a autoestima, maior será o respeito, benevolência e boa vontade com que tratamos os outros, pois não os percebemos como ameaça. Para ele "o autorrespeito é o fundamento do respeito pelos outros" (Branden, 1994: 11).

Outro conceito fundamental neste trabalho é o de cultura organizacional. Para este também é difícil encontrar uma definição única e abrangente. Schein (1992) destaca que a noção de cultura organizacional se insere em um conceito mais amplo de cultura. E pode ser definida como:

um padrão de pressupostos básicos que o grupo criou ou desenvolveu, aprendendo a lidar com os problemas de adaptação externa e integração interna, os quais funcionaram suficientemente bem, podendo assim ser ensinados aos novos membros como o modo correto de perceber, pensar e sentir em relação àqueles problemas (Schein, 1992: 7).

Pereira (1999: 289) considera que "tal como uma pessoa tem personalidade, a organização tem cultura”. A história da polícia brasileira, para Kant de Lima (1995), está impregnada de um elitismo judicial e de práticas judiciais discricionárias, frutos de uma contraditória ideologia igualitária e uma ordem social profundamente hierarquizada. Dessa forma, para compreender o conceito de cultura organizacional na instituição policial no Brasil, há que se ter em mente que em nosso país são dados diferentes tratamentos legais a infrações semelhantes, dependendo da situação socioeconômica do suspeito. Cabe então, inicialmente, compreender traços da cultura judiciária na qual a cultura da polícia está inserida, conforme reflete Kant de Lima (1995):

A polícia contamina suas funções de investigação pelas de vigilância. Em vez de apurar fatos, a polícia vigia a população, num processo preliminar de seleção para a aplicação da lei. O tratamento legal dispensado vai depender do status de cada pessoa [...]. Na realidade, a polícia prevê os fatos delituosos por meio de suposiçōes relativas ao caráter do deliquente [...] (Kant de Lima, 1995: 8).

Silva (2003) faz uma profunda reflexão acerca da influência dos aspectos culturais e das pressões nas polícias brasileiras. E afirma que, até os nossos dias, a violência continua a ser "calcada numa doutrina belicista" (Silva, 2003: 21), como 
no passado. Ele questiona como o policial pode respeitar os direitos humanos se é ensinado, nas próprias academias, a estar em estado constante de guerra e ver em cada cidadão um criminoso potencial. A partir dessas indagações, o autor denuncia que há uma "cultura da violência que permeia a sociedade brasileira" (Silva, 2003: 25). E a cultura organizacional da polícia sofre profundas influências desse estado de violência social.

Entende-se por cultura de violência, no caso específico da polícia, a normatização de atitudes violentas, abuso de poder, além da admissão de atuações desequilibradas por parte dos policias como corretas e normais (Adorno, 1999; Mesquita Neto, 1999). Segundo Silva (2003), para compreender essa cultura de violência, faz-se necessário considerar as profundas marcas autoritárias deixadas pelos " 500 anos de práticas repressivas, calcadas em nossa violência histórica” (Silva, 2003: 20). Pode-se inferir que o que faz a cultura organizacional da polícia ser tão identificada como uma cultura de violência, que desrespeita sistematicamente os direitos humanos, é o contexto em que ela se dá e as circunstâncias com que opera.

Wacquant (2001) pondera que essa forma violenta de operar está relacionada à pretensão de aumentar a força do Estado, coibindo o sentimento de insegurança causado, justamente, por menos Estado nas áreas econômica e social. Outro fator que complexifica ainda mais a situação da cultura de violência, de acordo com Wacquant (2000), é a importação do modelo de intransigência e repressão às pequenas infraçôes, cometidas em via pública, intitulado: "Tolerância zero". Este nasceu nos Estados Unidos (EUA), na década de 1990, em Nova York, na gestão de Rudolph Giuliani.

A adoção desse modelo de Estado Policial no Brasil acabou enveredando pela criminalização da pobreza. E tornou-se, ainda, um desrespeito ao Estado de Direito. Nesse sentido, Kant de Lima (1995) assegura que há dois posicionamentos antagônicos que a Polícia pode assumir: ser um instrumento da política de segurança do Estado, ou ser parte da política geral de direitos dos cidadãos.

Embora seja extremamente importante, a dificuldade de implantar e implementar um efetivo projeto de educação em direitos humanos existe. Segundo Silva (2003), isto se deve ao fato de que não basta transmitir informações sobre a história dos Direitos Humanos, a Constituição ou mesmo a Declaração Universal: o desafio fundamental, no caso policial, é "[...] mudar atitudes, comportamentos, quebrar paradigmas, enfim, promover uma mudança cultural” (Silva, 2003: 32).

Considerando o que foi exposto, o fornecimento de uma ação de suporte emocional, como estratégia de fortalecimento da autoestima, foi a intervenção utilizada. A intenção foi a mudança do clima e da cultura da delegacia onde se desenrolou esta pesquisa. 


\section{Metodologia}

Este estudo segue a orientação teórica da pesquisa-ação. Bulmer (1978) define pesquisa-ação como um tipo de pesquisa social, vinculando sua tipologia com a solução de um problema. Entretanto, ressalta que o sentido dessa modalidade de estudo é acompanhar o desenvolvimento de uma intervenção. Nesse sentido, buscou-se combinar o envolvimento dos pesquisadores com a "ação de intervenção", aplicando-se instrumentos quantitativos e qualitativos para medir e compreender os efeitos desse processo.

\section{PARTICIPANTES}

Participaram do estudo 148 policiais de uma delegacia especializada em crimes contra o patrimônio da cidade do Rio de Janeiro. Esses policiais foram alocados em dois grupos: experimental (GE) e controle (GC). Eles foram agrupados a partir de variáveis tais como: sexo, idade, tempo de serviço, tipo de funções (internas ou externas). O GE foi constituído por 76 componentes e o GC por 72 . Enquanto o GE participou da intervenção proposta nesta pesquisa, o GC fez um treinamento técnico oferecido pelo "Programa Delegacia Legal".

\section{INSTRUMENTOS}

$\mathrm{Na}$ abordagem quantitativa, um questionário autoaplicado foi preenchido pelos dois grupos antes (Fase 1) e após (Fase 2) a intervenção. Esse instrumento era composto por 76 questões, muitas delas provenientes das seguintes escalas: a Escala de Autoestima de Rosemberg e o Diagnóstico Organizacional (D.O.) de Rosa Krausz, que visa avaliar cultura organizacional.

A Escala de Autoestima de Rosemberg é formada por 10 questões fechadas em relação à satisfação pessoal e à atitude positiva ou negativa em relação a si mesmo. As questões são: (1) no conjunto, estou satisfeito comigo; (2) às vezes, eu acho que não presto para nada; (3) Eu sinto que eu tenho várias boas qualidades; (4) eu sou capaz de fazer coisas tão bem quanto a maioria das pessoas; (5) eu sinto que não tenho muito do que me orgulhar; (6) eu com certeza me sinto inútil, às vezes; (7) eu sinto que sou uma pessoa de valor, pelo menos do mesmo nível que as outras pessoas; (8) eu gostaria de poder ter mais respeito por mim mesmo; (9) 
no geral, estou inclinado a achar que sou um fracasso; (10) eu tenho uma atitude positiva com relação a mim mesmo.

As repostas são: concordo totalmente, concordo, discordo e discordo totalmente; e a pontuação de cada um dos itens varia de 1 a 4 pontos. No Brasil esta escala foi validada por Avanci, Assis, Santos, e Oliveira (2006).

A avaliação da autoestima foi feita por meio da escala supracitada e por outras questões isoladas relativas à satisfação com a profissão, aceitação pelos outros, estresse e sentimentos de depressão e projeto de futuro, cujos resultados serão aqui apresentados.

A cultura organizacional foi mensurada através do Diagnóstico Organizacional (DO) de Krausz (1994), que é um instrumento que avalia conjuntamente cultura e clima organizacional. Este é composto de 12 perguntas que faziam parte do questionário. Os resultados da escala de Rosa Krausz permitem categorizar o diagnóstico da cultura/clima da organização. Esta pontuação é dada pelas médias das respostas a cada uma das 12 dimensões (ver a tabela 1 em anexo).

As pontuações das médias de cada dimensão podem resultar em quatro tipos de cultura/clima que correspondem às seguintes alternativas: (1) organização caótica; (2) resistente a mudanças; (3) consciente da necessidade de mudanças e (4) aberta a mudanças.

A abordagem qualitativa utilizou um questionário aberto sobre a importância de cada encontro; um questionário aberto e um grupo de debate ao final da intervenção com a finalidade de avaliá-la; e um diário de campo com os registros dos facilitadores/pesquisadores e dinamizadores/convidados das oficinas sobre as impressões e pontos positivos e negativos das atividades. $\mathrm{O}$ tratamento desse material envolveu o processamento e a interpretação dos dados a partir do enfoque da análise temática, seguindo a perspectiva da análise de conteúdo de Bardin (1979), em sua versão adaptada por Minayo (2006).

A análise ocorreu em duas etapas. Na primeira, sistematizou-se a avaliação feita em cada oficina da intervenção segundo as informações dos policiais e dos pesquisadores. No segundo momento, foi feita a análise da avaliação final de todo o processo, também tendo como material o instrumento preenchido pelos policiais e o diário de campo dos pesquisadores.

Realizou-se a leitura flutuante e intensiva de todo o material. Em seguida foram organizados mapas, alocando-se todos os relatos pertinentes a cada temática em um mesmo arquivo. Os núcleos de sentido e suas autorias (se provenientes dos policiais ou dos pesquisadores) foram identificados e confrontados.

A intervenção, denominada de sensibilização vivencial, teve duração de 5 meses, em encontros quinzenais com duração de 3 horas cada, fora do local de 
trabalho. As oficinas foram conduzidas por especialistas em dinâmicas de grupo (dois facilitadores/pesquisadores), além de dez dinamizadores/convidados, especialistas nas temáticas abordadas (Andrade et al. 2009). Em algumas atividades o grupo experimental foi abordado por inteiro; em outras foi subdividido, ora em dois, ora em quatro subgrupos, objetivando maior aproximação entre participantes e desses com os especialistas.

Foram feitas dez oficinas com temas previamente discutidos e selecionados pela equipe de pesquisa e por consultores policiais: Integrando pela alegria I e II; Integrando para resgatar sonhos; Estresse e risco; Relaxando para aguentar o tranco; Polícia e vida familiar; Autoimagem do policial; Espiritualidade; Oficina de crescimento pessoal; Peça teatral Navegar é Preciso. Do total, seis privilegiaram a autoestima em suas abordagens.

Houve aprovação do Comitê de Ética da Escola Nacional de Saúde Pública Sergio Arouca, da Fiocruz, e todos os rigores de pesquisa foram seguidos.

\section{Resultados}

Segundo Andrade et al. (2009), o perfil dos policiais participantes da pesquisa tinha as seguintes características:

[...] 93\% por policiais do sexo masculino; $65 \%$ tinham 36 ou mais anos de idade; $64,2 \%$ se autodeclararam brancos; $50 \%$ possuíam curso superior completo e $4 \%$ tinham pós-graduação; a grande maioria estava casada ou tinha companheiro(a); 67,6\% referiram ter filhos; $42,6 \%$ entraram na instituição há até cinco anos e $36,5 \%$ tinham entre onze e vinte anos de trabalho na Polícia; 80,0\% ocupavam o cargo de inspetor de polícia (Andrade et al., 2009: 277).

De acordo com as respostas dadas no questionário, constata-se que a percepção dos policiais melhorou em relação à realização profissional após a sensibilização. Apesar de não haver diferença estatisticamente significativa, no início da pesquisa, visto que $68,4 \%$ dos policiais incluídos no grupo experimental se diziam satisfeitos profissionalmente; ao final da intervenção, 76,0\% passaram a pensar assim. Minayo e Souza (2003) encontraram uma proporção menor (53,3\%) de policiais civis que estavam satisfeitos com a realização profissional. Além disso, a maioria desses estava alocada no grupo técnico. Apenas 32,0\% dos lotados no setor administrativo se diziam satisfeitos $(32,0 \%)$.

Cerca de $41 \%$ dos policiais do grupo, sob intervenção, acreditam que existe uma visão positiva dos outros em relação a eles. Suas respostas não apresentaram 
diferença estatisticamente significativa entre as fases 1 e 2 . No grupo controle, as proporçôes foram maiores e crescentes ao longo do estudo (43,7\% no início e $47,8 \%$ na fase 2 ).

A influência que o estresse no trabalho tem sobre as relações dos policiais foi aqui investigada e apresentou uma mudança positiva no grupo experimental, quando comparada com o grupo controle. $\mathrm{Na}$ fase 1 da pesquisa $51,3 \%$ deles informavam que o estresse no trabalho se refletia nos seus relacionamentos familiares e com os amigos. $\mathrm{Na}$ fase 2 apenas $38,8 \%$ fizeram tal afirmação. O mesmo não aconteceu com o grupo controle. No início os que concordavam com essa assertiva eram $43,0 \%$ e no final da pesquisa foram $52,2 \%$ deles, como se pode ver no gráfico 1 (ver em anexo).

Todas essas questôes - realização profissional, ser bem visto pelas outras pessoas e o estresse do trabalho - influenciam as expectativas que esses policiais têm em relação à profissão de seus filhos. Embora a maioria deles se considere satisfeita com a realização profissional, apenas metade afirma que estimularia seus filhos à carreira de policial civil, caso esse fosse o desejo deles.

Os resultados da escala de autoestima demonstraram que houve mudanças significativas, no grupo experimental, da fase 1 para a fase 2 . No grupo de controle, não se perceberam mudanças significativas da autoestima após a intervenção (Andrade et al., 2009).

Os resultados do Diagnóstico Organizacional de Rosa Krausz demonstraram que, numa avaliação global dos itens desse instrumento, no Grupo Experimental as respostas transitaram das alternativas 3 para as de número 4, quando se comparam as fases 1 e 2 . Em outras palavras, a corporação que anteriormente era percebida como consciente da necessidade de mudanças passa a ser vista como mais aberta às mudanças.

A análise qualitativa mostrou que algumas falas dos policiais, nas avaliações das oficinas, foram coerentes com os dados do questionário, ao verbalizarem sentimentos de felicidade: "me sinto mais leve, feliz, depois da palestra"; ou ainda que "atividades como essa aumentam a vontade de viver!".

Questôes relacionadas à necessidade de maior união e corporativismo da classe, visando a uma mudança na imagem do policial, também foram mencionadas. Chama atenção a fala de um dos integrantes a respeito das oficinas: "a atividade é um incentivo a uma autoavaliação profunda quanto ao caminho a ser seguido, trilhado, para alcançar a ética profissional necessária ao bom desempenho do policial civil”.

Com o intuito de avaliar o processo como um todo, foram feitas as seguintes perguntas aos policiais: (1) em que a sensibilização contribuiu para a sua vida 
profissional e pessoal; (2) se percebiam alguma diferença entre essas atividades e outras de que tenham participado na polícia; pontos fortes e fracos e comentários sobre toda a intervenção.

Nos 45 questionários respondidos, a maioria dos policiais (35) avaliou que a intervenção contribuiu tanto para a vida pessoal quanto profissional. No entanto, alguns agentes (nove) registraram que as ações pouco ou nada contribuíram para suas vidas. As contribuições mais mencionadas por eles foram: aprender a lidar com as adversidades; a integração da equipe; a possibilidade de reflexão sobre a qualidade de vida no âmbito pessoal e profissional; e a minimização do estresse (pelo relaxamento e humor).

\section{CONSIDERAÇŌES FINAIS}

Este trabalho buscou demonstrar que atuar na autoestima, além de possibilitar mais saúde mental, pode ser um dispositivo estratégico para suscitar mudanças na cultura de violência vivenciada nas polícias das grandes metrópoles do nosso país. Como afirma Balestreri (2003: 1), "a elevação dos padrões de autoestima pode ser o caminho mais seguro para uma boa prestação de serviços na polícia”. Este autor ainda vai pontuar que um dos requisitos básicos para se chegar a querer bem ao outro é querer-se bem. Foi nesta direção que a intervenção procurou trabalhar.

Percebeu-se que os seus efeitos ocorreram em diferentes intensidades, mas, de maneira geral, boa parte dos policiais considerou o projeto relevante. Declararam, ainda, que, em todo o tempo de polícia, nunca haviam tido oportunidade de discutir estes temas de forma sistemática. Uma constatação relevante é que a pesquisa proporcionou a criação de um espaço de escuta e troca entre facilitadores e policiais ao longo dos cinco meses de intervenção.

Silva (2003) afirma que a educação em direitos humanos para as polícias brasileiras é o principal veículo para democratizar a Segurança Pública no país. A sensibilização vivencial empreendida pode alcançar bons resultados nessa direção, uma vez que constitui metodologia aplicável a grupos similares (policiais militares, forças armadas, bombeiros e outros) cuja missão é garantir a segurança pública mas não exercem plenamente esse papel pelos diversos atravessamentos a que estão sujeitos. Entretanto, Balestreri (2003) acrescenta que a formação dos operadores dos diretos de segurança pública e a busca de melhores condiçôes de vida para esses profissionais ainda tem um longo caminho a ser percorrido.

Veloso (2008), em seu livro É possivel, discorre sobre a exemplar experiência ocorrida no morro Dona Marta, na cidade do Rio de Janeiro, em que 
uma intervenção da polícia, que leva em conta os direitos dos cidadãos, pode ser transformadora. Esta pesquisa, diferente do que muitos afirmam, alinha-se a iniciativas que acreditam que, mesmo no Rio de Janeiro, se pode ter uma polícia melhor.

Finalmente, é importante realçar dois importantes méritos do presente estudo: (1) ser uma contribuição dentre as poucas pesquisas com policiais em nossa realidade; (2) constituir-se numa intervenção sistematizada com esses profissionais entre um número extremamente reduzido de estudos dessa natureza.

\section{REFERÊNCIAS}

Adorno, S. (1999). Violência e civilização. In: Santos, J. V. \& Gugliano, A. A. (Orgs.). A sociologia para o céc. XXI. Pelotas: Educat.

Andrade, E. R., Sousa, E. R. \& Minayo, M. C. S. (2009). Intervenção visando a autoestima e qualidade de vida dos policiais civis do Rio de Janeiro. Ciênc. saúde coletiva, 14(1). Disponível: <http://www.scielo.br/scielo.php?script=sci_arttext\&pid=S1413$81232009000100034 \& \operatorname{lng}=$ pt\&nrm=iso $>$.

Avanci, J. Q., Assis, S. G., Santos, N. C. \& Oliveira, R. V. C. (2006). Adaptação transcultural de Escala de Auto-Estima para Adolescentes. [ site na internet] disponível em <http://www. scielo.br/pdf/v20n3/a07v20n3.pdf>.

Bardin, L. (1979). Análise de conteúdo. Lisboa: Edições 70.

Bulmer, M. (1978). Social policy research. Londres: The Mcmillan Press Ltd.

Balestreri, R. B. (2003). Direitos Humanos: coisa de polícia. Passo Fundo: Ed. CAPEC.

Branden, N. (1994). Auto-Estima: como aprender a gostar de si mesmo. São Paulo: Editora Saraiva.

Branden, N. (1995). Auto-Estima e os seus seis pilares. São Paulo: Editora Saraiva.

Dejours, C. (1999). O fator humano. Rio de Janeiro: Fundação Getúlio Vargas.

Dolan, S. (2006). Estresse, auto-estima, saúde e trabalho. Rio de janeiro: Qualitmark.

Gaulejac, V. (2006). As origens da vergonha. São Paulo: Via Lettera Editora e Livraria.

James, W. (1885/1974). Os pensadores. São Paulo: Abril Cultural.

Kant de Lima, R. (1995). A polícia da cidade do Rio de Janeiro: seus dilemas e paradoxos. Rio de Janeiro: Forense.

Krausz, R. R. (1994). Diagnóstico organizacional: forma I e II. São Paulo: Casa do Psicólogo.

Mesquita Neto, P. (1999). Violência policial no Brasil: abordagens teóricas e práticas de controle. In: Pandolf, D. C, Carvalho, J. M., Carneiro, L. P. \& Grynszpan, M. (Orgs.). Cidadania, justiça e violência. Rio de Janeiro: Fundação Getúlio Vargas. 
Minayo, M. C. S. (2006). O desafio do conhecimento (9a edição). São Paulo: HUCITEC. Minayo, M. C. S. \& Souza, E. R. (2003). Missão investigar-Entre o ideal e a realidade de ser policial. Rio de Janeiro: Garamond.

Njaine, K., Assis, S. G., Ribeiro, F. M. L., Oliveira, Q. B. M., Souza, F. A., Oliveira, R. V. C., Marriel, L. C, Njaine, B. \& Motta, D. M. (2006). A representação do policial na mídia escrita e seu significado na violência - Estudo comparativo de quatro capitais brasileiras. In: Anais do $11^{\circ}$ Congresso Mundial de Saúde Pública e $8^{\circ}$ Congresso Brasileiro de Saúde Coletiva, Rio de Janeiro.

Pereira, O. G. (1999). Fundamentos de comportamento organizacional (2o ed.). Lisboa: Fundação Calouste Gulbenkian.

Schein, E. H. (1984). Coming to a new awereness of organizational culture. Sloan Management Review, 25, (2).

Schein, E. H. (1992). Organizational culture and leadership. San Francisco: Jossey-Bass.

Silva, S. S. (2003). Teoria e prática da Educação e Direitos Humanos nas instituições policiais brasileiras. Porto Alegre: Edições Capec.

Silveira Filho, S. L. D. (2007). Neoliberalismo, midia e movimento de lei e ordem: rumo ao Estado de Polícia. Rio de Janeiro: Editora Revan/ICC.

Staerke, R. (1996). Auto-estima em psicologia, uma proposta de definição. Rio de Janeiro: UFRJ, Instituto de Psicologia.

Veloso F. (2008). É possível - gestão da segurança pública e redução da violência. Rio de Janeiro: Contra-Capa Editora.

Wacquant, L. (2000). Punir os pobres: a nova gestão da miséria nos Estados Unidos. Rio de Janeiro: ICC/Freitas Bastos.

Wacquant, L. (2001). As prisóes da miséria. Rio de Janeiro: Jorge Zahar.

\section{Anexo}

Tabela I. Diagnóstico Organizacional de Rosa Krausz

\section{Relaçóes entre as pessoas:}

I. $\square$ RARAS: AS PESSOAS PERMANECEM, SEMPRE QUE POSSÍVEL, ISOLADAS UMAS DAS OUTRAS.

2. $\square$ LIMITADAS: As PESSOAS FORMAM GRUPINHOS FECHADOS PARA SE PROTEGEREM.

3. $\square$ APOIADORAS: As PESSOAS SE APROXIMAM E SE ABREM COM QUEM AS APÓIA.

4. $\square$ ABERTAS: TODOS SE SENTEM ACEITOS E SABEM QUE DEPENDEM UNS DOS OUTROS. 


\section{Padróes de relacionamentos mais comuns:}

I. $\square$ NECESSÁRIOS: AS PESSOAS SE RELACIONAM O MÍNIMO NECESSÁRIO PARA MANTEREM SEU EMPREGO.

2. $\square$ DE INTERESSE: As PESSOAS SÓ SE RELACIONAM QUANDO HÁ INTERESSE DE MOMENTO.

3. $\square$ CONFIÁVEIS: As PESSOAS SE RELACIONAM COM AQUELES EM QUEM CONFIAM.

4. $\square$ CONJUNTOS: TODOS SE RELACIONAM FACILMENTE.

\section{Relaçóes entre grupos:}

I. $\square$ OCASIONAIS: O RELACIONAMENTO ENTRE ÁREAS É RARO.

2. $\square$ DE TROCA: As Áreas SE RELACIONAM PARA ATENDER aOS SEUS INTERESSES.

3. $\square$ De ColaboraÇÃo: As PESSOAS SE REUNEM PARA RESOlVER PROBLEMAS COMUNS.

4. $\square$ COOPERATIVAS: As ÁREAS COOPERAM, TENDO EM VISTA OS INTERESSES DA INSTITUIÇÃO.

\section{Comunicação:}

I. $\square$ DEFICIENTE: A COMUNiCAÇÃo É OCASIONAL E INCOMPleta. FALTAM INFORMAÇÕES IMPORTANTES.

2. $\square$ LIMITADA: A COMUNICAÇÃO OBEDECE ÀS NORMAS EXISTENTES.

3. $\square$ SATISFATÓRIA: As INFORMAÇÕES IMPORTANTES SÃO TRANSMITIDAS COM DETALHES.

4. $\square$ BOA: As INFORMAÇÕES SÃO PASSADAS PARA TODOS NA INSTITUIÇÃO.

\section{Canais de Comunicação:}

I. $\square$ INTERROMPIDOS: POUCA COMUNICAÇÃO ENTRE AS PESSOAS E NÍVEIS HIERÁRQUICOS.

2. $\square$ Semifechados: Predomina a COMUniCaÇÃo formal ENTRE CHEFES E os SUBORDINADOS E ENTRE AS PESSOAS DE MESMO NÍVEL.

3. $\square$ SEMI-ABERTOS: NAS INFORMAÇÕES DE ROTINA, A COMUNICAÇÃO FORMAL É MAIS FREQUENTE. HÁ MUITOS BOATOS E FOFOCAS.

4. $\square$ ABERTOS: A COMUNICAÇÃo É CONSTANTE ENTRE OS NÍVEIS HIERÁRQUICOS E ENTRE AS PESSOAS DO MESMO NÍVEL.

\section{Estilos de Liderança:}

I. $\square$ OMISSO: CAdA UM FAZ O QUE QUER. NÃo HÁ COORDENAÇão de ATIVIdAdes/ AÇÕES.

2. $\square$ AUTORITARIO: MANDA QUEM PODE, OBEDECE QUEM TEM JUÍZO.

3. $\square$ CONSUltiVo: AS OPINIÔES SÃO SOliCiTADAS, MAS É A CÚPUla QUE DECIDE.

4. $\square$ PARTICIPATIVO: TOdos OS INTERESSADOS PARTICIPAM DAS DECISÕES QUE ENVOLVEM SUAS ÁREAS. 


\section{Tomada de Decisão:}

I. $\square$ DESORDENADA: As DECISÕES SÃo TOMADAS DE ACORDO COM O QUE ACONTECE, SEM OCORRÊNCIA OU SISTEMATICA.

2. $\square$ CENTRALIZADA: As DECISÕES SÃO TOMADAS COM BASE EM CRITÉRIOS PRÉ-ESTABELECIDOS E INFLEXÍVEIS.

3. $\square$ COMPARTILHADA: O PROCESSO DECISÓRIO BASEIA-SE EM CONSUlTAS ÀS DIFERENTES ÁREAS.

4. $\square$ INTEGRADA: As DECISÕES SÃO TOMADAS DE MANEIRA FLEXÍVEL, CONSIDERANDO-SE A EMPRESA COMO UM TODO.

\section{Planejamento:}

I. $\square$ INEXISTENTE: NÃo HÁ PLANEJAMENTO, APENAS IMPROVISO.

2. $\square$ POR CRISE: Só SE Planeja PARA ENFRENTAR CRISES.

3. $\square$ Parcial: Planeja-se apenas dados já existentes e disponíveis.

4. $\square$ Sistemático: PlaneJa-Se COM Base EM Dados CONfiáveis, atualizados E ADEQUADOS.

\section{Soluçáo de problemas:}

I. $\square$ OCASIONAL: OS PROBLEMAS ACUMULAM-SE E POUCOS SÃO RESOLVIDOS.

2. $\square$ Tradicionalista: Preferem-Se Soluçôes tradicionais ou técnicas CoNHECIDAS, MESMO NÃO FUNCIONANDO.

3. $\square$ OPORTUNISTAS: OS PROBLEMAS SÃo SOlUCIONADOS SEMPRE QUE SURJE UMA OPORTUNIDADE PARA FAZÊ-LO.

4. $\square$ ORDENADA: ACOMPANHA-SE O QUE ESTÁ ACONTECENDO PARA PROCURAR SOLUÇÃO E EVITAR PROBLEMAS.

\section{Trabalho em equipe:}

I. $\square$ INEXISTENTE: CADA UM TRABALHA POR SI.

2. $\square$ POUCO FREQUENTE: Às VEZES TRABALHA-SE EM EQUIPE, APESAR DA FALTA DE INTEGRAÇÃO DAS PESSOAS, PREJUDICANDO SUA PRODUTIVIDADE.

3. $\square$ Limitado: TrabalHa-SE EM EQUipe, APESAR DA FALTA DE INTEgRaÇÃo Das PESSOAS, PREJUDICANDO SUA PRODUTIVIDADE.

4. $\square$ OPÇÃO: TRABALHA-SE DE FORMA INTEGRADA, HAVENDO COOPERAÇÃO E ACEITAÇÃO DE TODOS. 


\section{Clima da empresa:}

I. $\square$ CAÓTICO: As PESSOAS SE SENTEM INSEGURAS, INSATISFEITAS E DESCONHECEM A EMPRESA.

2. $\square$ DEPENDENTE: As PESSOAS EXECUTAM TAREFAS E NÃO RECONHECEM OS OBJETIVOS DA EMPRESA COMO UM TODO.

3. $\square$ VARIÁVEL: As PESSOAS NEM SEMPRE ESTÃo SATISFEITAS COM A SITUAÇÃO, MAS NÃO SABEM O QUE FAZER PARA MUDÁ-LA.

4. $\square$ SAUDÁVEL: As Pessoas estão SATISFEITAS COM A SitUAÇÃo PRESENTE, MaS NÃO TEM MEDO DAS MUDANÇAS.

\section{Motivação:}

I. $\square$ NÃO EXISTENTE: As PESSOAS PERMANECEM ISOLADAS DA REALIDADE E NÃO RECEBEM INFORMAÇÕES SOBRE O SEU DESEMPENHO.

2. $\square$ BAIXA: As PESSOAS CUMPREM SUAS ROTINAS. CRITICA-SE MUITO E AS MUDANÇAS NÃO SÃO BEM ACEITAS.

3. $\square$ MÉdia: As SUgESTÕ̂s de MUdANÇA SÃo aCEITAS, MAS AS PESSOAS PROCURAM FICAR NA ROTINA. Às VEZES AS PESSOAS SÃo ELOGIADAS.

4. $\square$ ALTA: As PESSOAS ESTÃo SEMPRE dispostas a ACEITAR NOVOS DESAFios. ReCONHECE-SE O VALOR E A COMPETÊNCIA DE CADA UM.

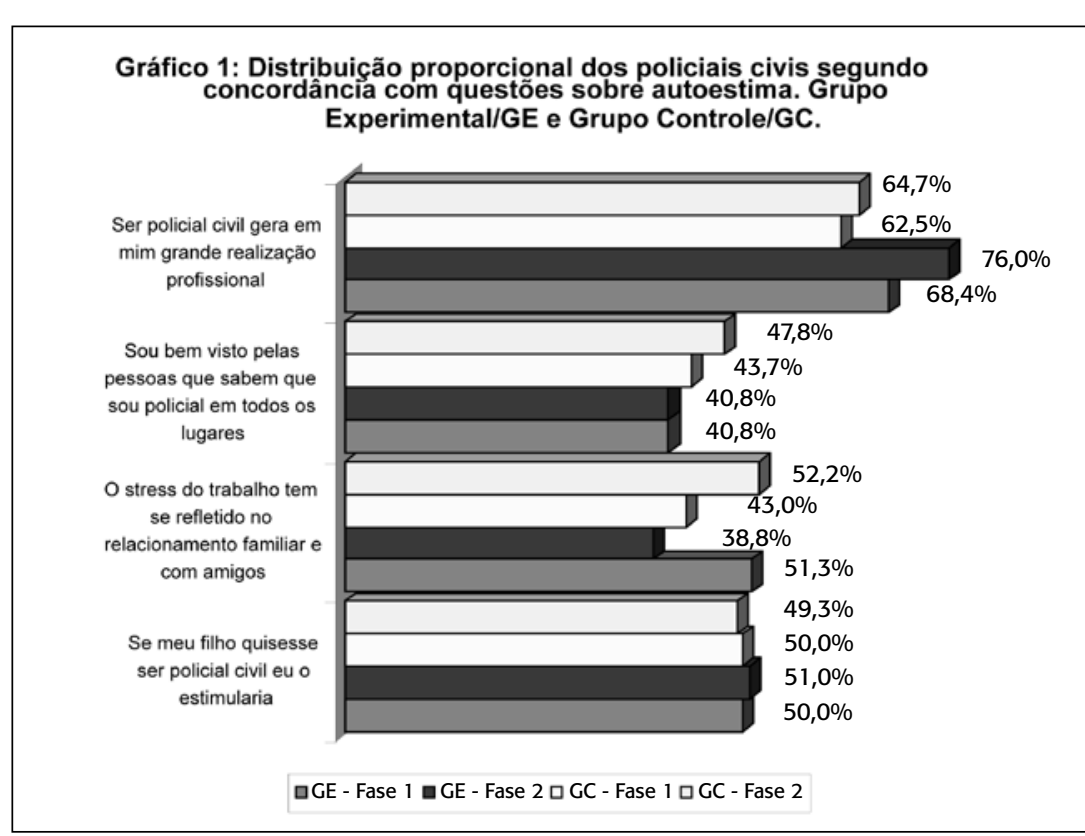

Recebido em 19 de janeiro de 2009

Aceito para publicação em 18 de outubro de 2010 